Wolf-Rayet Phenomena in Massive Stars and Starburst Galaxies

Proceedings IAU Symposium No. 193, (C)1999 IAU

K.A. van der Hucht, G. Koenigsberger \& P.R.J. Eenens, eds.

\title{
The superwind Wolf-Rayet galaxy Markarian 1259
}

\author{
Youichi Ohyama and Y. Taniguchi \\ Astronomical Institute, Tohoku University, Japan \\ Robert J. Terlevich \\ Institute of Astronomy, University of Cambridge, UK
}

\begin{abstract}
We present results of our optical spectroscopic observations and ${ }^{12} \mathrm{CO}(J=1-0)$ mapping of the Wolf-Rayet starburst galaxy Markarian 1259 with a superwind activity. We find that both the ionized gas and the molecular gas are associated with the superwind.
\end{abstract}

The pole-on viewed superwind of the Wolf-Rayet galaxy Markarian 1259 is investigated both by optical spectroscopy and by ${ }^{12} \mathrm{CO}(J=1-0)$ mapping. The optical spectrum at the nuclear region shows a prominent Wolf-Rayet feature around $4650 \AA$, indicating the presence of 4100 WRL stars (Ohyama, Taniguchi \& Terlevich 1997). We find that the shock-excited ionized gas is spatially extended over a $\sim 2.3 \mathrm{kpc}$ region, suggesting the presence of a superwind. The stronger [NII] emission indicates the over-abundance of nitrogen.

The ${ }^{12} \mathrm{CO}(J=1-0)$ emission is detected in the nuclear star-forming region and even in the off-nuclear regions ( $\sim 2 \mathrm{kpc}$ apart from the nucleus). Since the velocity field of the off-nuclear molecular gas cannot be explained by a model of a simple disk rotation, we suggest that the molecular gas is associated with the superwind (Ohyama \& Taniguchi 1998). Our recent high-resolution ${ }^{12} \mathrm{CO}$ $(J=1-0)$ map obtained with the Nobeyama Millimeter Array $(N M A)$ of this galaxy, has shown clearly that there are three velocity components in the offnuclear regions: a disk component at the systemic velocity and two high-velocity components (red-shifted and blue-shifted ones). This kind of velocity field is well represented by our simple model of pole-on biconical outflow in which the molecular gas is blowing off the nucleus (Ohyama \& Taniguchi 1998, 1999).

\section{References}

Ohyama, Y., Taniguchi, Y. 1998, ApJ 498, L27

Ohyama, Y., Taniguchi, Y. 1999, in preparation

Ohyama, Y., Tanigchi, Y., Terlevich, R. 1997, ApJ 480, L9 